\title{
Trazando rutinas: el papel del ferrocarril en las prácticas cotidianas y movilidades interurbanas de habitantes de La Araucanía, Chile
}

\author{
Traçando rotinas: o papel da ferrovia nas práticas cotidianas e na mobilidade
} interurbana dos habitantes de La Araucanía, Chile

Tracing routines: the role of the railway in daily practices and interurban mobilities of the inhabitants of La Araucanía, Chile

\section{Hernán Riquelme Brevis[a,b] (D) , Alejandra Lazo Corvalán[c]}

[a] Universidad Autónoma de Chile, Temuco, Chile

[b] Universidad Arturo Prat, Victoria, Chile

[c] Universidad de Los Lagos, Centro de Estudios de Desarrollo Regional y Políticas Públicas (CEDER),

Osorno, Chile

Cómo citar: Brevis, H. R., \& Corvalán, A. L. (2019). Trazando rutinas: el papel del ferrocarril en las prácticas cotidianas y movilidades interurbanas de habitantes de La Araucanía, Chile. urbe. Revista Brasileira de Gestão Urbana, 11, e20180034. https://doi.org/10.1590/2175-3369.011.002.A002

\section{Resumen}

El presente artículo analiza las prácticas de movilidad cotidiana de los usuarios del ferrocarril de La Araucanía en el sur de Chile. A través de un enfoque etnográfico fue posible explorar dos experiencias de habitantes de la región que por motivos laborales (conmutación) viajan cotidianamente a una ciudad distinta de aquella en la que residen, permitiéndoles conocer diversos medios de transporte, espacios de vida y lugares de frecuentación desde una perspectiva experiencial. Dentro de los hallazgos, cabe destacar la relevancia del ferrocarril como dispositivo de uso frecuente en la conexión, acceso, participación e inclusión en los diversos ámbitos de la vida cotidiana, generando una distinción a nivel simbólico y material respecto a otros medios de transporte público y formas de movilidad. Finalmente, fue posible dar cuenta de la existencia de una movilidad interurbana que transgrede la planificación estática y tradicional, abriendo interrogantes respecto a cómo se experimentan los límites del espacio habitado en las ciudades intermedias y en las poblaciones del sur de Chile.

Palabras clave: Movilidad ferroviaria. Inclusión social. Conmutación. Sur de Chile.

\section{Resumo}

O artigo analisa as práticas cotidianas de mobilidade dos usuários da ferrovia de La Araucanía no sul do Chile. Através de uma abordagem etnográfica, foi possivel explorar duas experiências de habitantes da região que, por razões de trabalho (comutação), viajam diariamente para uma cidade diferente daquelas em que moram, permitindo-lhes conhecer diferentes meios de transporte, espaços de vida e lugares de frequência, a partir de uma perspectiva experiencial. Entre as descobertas, é importante destacar a relevância da ferrovia como um dispositivo de uso frequente na conexão, acesso, participação e inclusão nas diversas áreas da vida cotidiana, gerando uma distinção simbólica e material em relação a outros meios de transporte público e formas de mobilidade. Finalmente, foi possível registrar a existência de uma mobilidade interurbana que transgride o planejamento estático e

HRB es doctor en Ciencias Sociales, e-mail: h.riquelmebrevis@gmail.com

ALC es doctora en Geografía y Planificación Territorial, e-mail: alejandra.lazo@ulagos.cl 
tradicional, abrindo questionamentos sobre como os limites do espaço habitado são vivenciados nas cidades intermediárias e povoados do sul do Chile.

Palavras-chave: Mobilidade ferroviária. Inclusão social. Comutação. Sul do Chile.

\section{Abstract}

This article examines everyday mobility practices of railway users of La Araucanía, south of Chile. Through an ethnographic approach it was possible to explore two experiences of inhabitants of this region that due to work reasons (commuting) travel daily from the city where they live to another, being able to know different means of transport, lifestyle spaces and common places from an experiential perspective. Among the findings, it should be pointed out the relevance of the railway as a device of common use, participation and inclusion in the different dimensions of everyday life, which generates a symbolic and material distinction in relation to other means of public transport and forms of mobility. Finally, it was possible to shed light on the existence of an interurban mobility that oversteps the static and traditional planning, opening questions regarding the way in which the limits of the inhabited space in intermediate cities and areas of the south of Chile are experienced.

Keywords: Railway mobility. Social inclusion. Commuting. South of Chile.

\section{Introducción}

La movilidad cotidiana constituye un objeto y enfoque de investigación con larga data en las ciencias sociales. Si durante la década de los noventa se tiende a consolidar el paradigma de la movilidad producto de una propuesta teórica que cada vez presta más atención a las interacciones sociales urbanas de la población en un contexto dinámico (Urry, 2007; Cresswell, 2006; Sheller \& Urry, 2006), con anterioridad a este giro epistemológico arraigado principalmente en los estudios anglosajones, se encuentran relevantes investigaciones que ponen en el centro del análisis las prácticas de movilidad. Como ejemplo de ello, Simmel (2005), identifica el papel de los estímulos de la ciudad en los urbanitas y cómo estos generaban asignaciones psicosociales específicas al desplazamiento, con ciertas actitudes que permitían afrontar la vida cotidiana. Asimismo, en las décadas de los sesenta y setenta, diversas corrientes de pensamiento reflexionaban respecto a la migración campo/ciudad, como proceso constitutivo de la movilidad y el territorio (Singer, 1975; Castells, 1973).

La movilidad, anclada en campos analíticos cuyas implicancias recaen en aspectos económicos y culturales de una sociedad caracterizada por el desarrollo tecnológico y la globalización capitalista (Ramírez, 2013), deviene en una práctica inherente del ser humano, de tiempos remotos y con una connotación actual única que permite considerar la relación entre espacio-tiempo y el desarrollo de las actividades rutinarias, sin dejar de advertir aspectos macrosociales que inciden en las subjetividades móviles, como medios de transporte, planificación urbana, lugar de residencia, entre otros.

En la actualidad latinoamericana, diversos estudios han puesto en el centro de análisis- y la discusión pública- la necesidad de explorar las transformaciones socioterritoriales a partir de las experiencias de movilidad en las metrópolis (Jirón et al., 2010; Segura, 2006; Avellaneda, 2008; Rolnik \& Klintowitz, 2011; Aguiar, 2011; Soto, 2017; Lazo-Corvalán \& Calderón-Sosa, 2013, 2014), encontrando un panorama complejo respecto a las diferencias sociales que se exacerban producto de aspectos socioeconómicos, infraestructura vial, concentración de servicios, marginación y desigualdades de género, por nombrar algunas de las tantas categorías de análisis que reflejan las prácticas de movilidad en ciudades caracterizadas por la desigualdad social.

Considerando que los estudios de la movilidad cotidiana en metrópolis latinoamericanas evidencian consecuencias adversas para algunos grupos compuestos principalmente por mujeres, ancianos, niños y sectores empobrecidos económicamente (Cosacov, 2015; Gutiérrez, 2009; Jouffe \& Lazo-Corvalán, 2010), el presente trabajo ahonda, por un lado, en las experiencias de movilidad no metropolitana en Chile 
(Lazo-Corvalán \& Ther, 2014; Lazo-Corvalán \& Carvajal, 2018), como área de incipiente investigación, por ende, con un alto grado de vacancia para problematizar respecto a los estudios de movilidad en territorios caracterizados por la relación económica y cultural entre ciudades medianas y pequeñas. Por otro lado, se exploran los límites espaciales y temporales de la movilidad interurbana desarrollada por usuarios del ferrocarril, que construyen y planifican las actividades cotidianas en espacios de frecuentación compuestos por diversos lugares que no se encuentran necesariamente en la ciudad de residencia, por consiguiente, el viaje se torna una actividad de primer orden, permitiendo indagar en las estrategias de movilidad que despliegan para desarrollar las actividades cotidianas.

El artículo se compone de cinco secciones. En la primera, se entregan reflexiones teóricas respecto al papel de la movilidad en los estudios vinculados a las diferencias sociales contraídas en la ciudad. En la segunda sección se describe el contexto general respecto a La Araucanía, considerando aspectos socioeconómicos y prácticas de desplazamiento. En la tercera parte del artículo se presenta la metodología empleada en el estudio. En la cuarta sección se exhibe la sistematización del trabajo de campo. Finalmente, se genera una discusión para pensar la movilidad en clave interurbana y en relación con el ferrocarril como dispositivo de primer orden en La Araucanía.

\section{Movilidades diversas y constantes}

El enfoque que nos interesa utilizar para el estudio de la movilidad de los usuarios del ferrocarril anclado en las prácticas sociales pone en el centro de análisis las experiencias de los sujetos en la vida cotidiana por sobre descripciones que reflejen cantidades de viajes o estructuras que inciden en el desarrollo de la movilidad. Esta perspectiva, de raíz fenomenológica, permite explorar diversas dimensiones de la movilidad y prestar atención a grupos de personas con características socioculturales y económicas particulares. Por consiguiente, comprender el significado y el movimiento que los viajeros otorgan a las experiencias conllevará a considerar, desde la masividad de la movilidad, las prácticas y las rutinas móviles (Cresswell, 2010; Jirón \& Mansilla, 2014).

Diversos estudios han pesquisado la movilidad de grupos sociales con necesidades específicas, ejemplo de ello son las investigaciones respecto a la movilidad de adultos mayores (Stjernborg, 2017; Schwanen \& Páez, 2010); las experiencias de niños y niñas en la ciudad (Cortés, 2011; Fagerholm \& Broberg, 2011); y, las diferencias de género en el espacio público y privado (Jirón, 2007; Jirón \& Zunino, 2017). Algunos de los hallazgos abren el debate con relación a las múltiples diferencias entre el ideal de ciudad y la ciudad real.

Estas contradicciones, suscitadas por desigualdades sociales que promueven escenarios de exclusión social, vulnerabilidad económica y desconexión urbana, conllevan a plantearse la capacidad de ser móvil, lo cual involucra desarrollar prácticas sociales de desplazamiento diario a través del tiempo y espacio que permite el acceso a diversas actividades, personas y lugares (Jirón, et al., 2010); practicar una movilidad reducida, lo cual involucra desarrollar la movilidad bajo ciertas limitaciones de carácter físico y emocional que inciden en el (des)conocimiento de la ciudad habitada; o lisa y llanamente la inmovilidad. Esta última "[...] podría llegar a ser una importante fuente de desigualdades y de nuevas conflictividades sociales" (Landon, 2013, p. 5).

No obstante lo anterior, existe la posibilidad de incluir en el análisis diversas maneras y formas de experimentar la ciudad desde la triada movilidad urbana, anclaje en la proximidad e inmovilidad (Lazo-Corvalán \& Calderón-Sosa, 2013, 2014). La movilidad incide en todas las esferas de la vida cotidiana, no solo en lo relativo al desplazamiento o la conexión de diversos lugares, esto conlleva a aumentar la complejidad analítica donde el lugar de residencia adquiere un papel fundamental en la participación social y el desarrollo de las prácticas de movilidad, considerando los factores del entorno y las experiencias personales que inciden en el acceso a personas y actividades 
diversas. No se puede ser constantemente móvil, reducidamente móvil o inmóvil, más bien se transita entre unos estados y otros según diversos factores asociados al ciclo vital, nivel socioeconómico, condiciones culturales, lugar de residencia o medios de transporte utilizados.

En el presente siglo, la movilidad virtual constituye un campo de interés que cada vez cobra mayor importancia en los estudios de la ciudad (Ribera Fumaz et al., 2007). También, las maneras de moverse han cambiado producto de la inserción de nuevos dispositivos de transporte en diversos periodos de la historia, impactando así en las experiencias de viaje metropolitano (Errázuriz, 2012). Las transformaciones culturales y económicas en las experiencias de viaje evidencian que la movilidad requiere de varias lecturas respecto a cómo vive y se relaciona el sujeto en la sociedad contemporánea.

Los estudios que han analizado las condiciones personales en el movimiento de los sujetos indican que los circuitos de transporte inciden directamente en las barreras de accesibilidad urbana (Avellaneda, 2008; Jouffe \& Lazo-Corvalán, 2010), reflejando los escasos esfuerzos políticos por hacer de la ciudad un espacio inclusivo, democrático y participativo, especialmente para los sectores sociales que viven en la marginalidad. Los derechos para crear y decidir qué tipo de ciudad se quiere (Lefebvre, 1975), manifiesta que la participación es una dimensión problemática y multifactorial. Para Harvey (2012), la privatización sostenida de los medios sociales pone en estado de alerta la creación de una esfera pública participativa y con espacios urbanos comunes. La crítica a la sociedad neoliberal resulta el primer eslabón respecto a las barreras que merman la participación y la accesibilidad de algunos por sobre otros en la ciudad.

Para Jirón \& Mansilla (2014, p. 59), la accesibilidad tiene relación con “[...] la habilidad de negociar el tiempo y el espacio para cumplir con actividades cotidianas, mantener relaciones y generar los lugares que las personas necesitan para participar en sociedad". Aunque los autores manifiestan que esta perspectiva se asemeja a un análisis con enfoque en la conexión/desconexión, abre la interrogante respecto a cómo las necesidades (básicas o elevadas) son disimiles entre los sujetos más allá de los deseos expresados. Algunas de estas diferencias estarían dadas por el habitar, la heterogeneidad de motivaciones o condiciones socioculturales.

En consecuencia, la relación entre participación y accesibilidad está dada por diversos factores sociales que encuentran niveles de relación entre el sujeto y la red socio-urbana que opera en el desarrollo de la movilidad cotidiana, movilidad reducida o una aparente inmovilidad. Entre los matices existentes, el dispositivo de transporte cobra valor no sólo por la funcionalidad, sino que, como se verá más adelante, por el valor agregado que los usuarios, a través de lógicas comparativas, añaden a este.

\section{Conmutación y exclusión socioespacial en La Araucanía}

Temuco, capital de La Araucanía, se encuentra a 680 kilómetros al sur de la ciudad de Santiago, ubicándose en la novena posición respecto a las comunas con más habitantes del país, con un total de 290.234 (INE, 2016). Temuco, concentra la mayor cantidad de servicios, entre los cuales destacan universidades, comercio, centros de salud, escuelas, instituciones públicas, entre tantos otros que obligan a que una gran cantidad de los habitantes de la región que no residen allí se desplacen cotidianamente con el objetivo de asistir a los servicios demandados.

La Araucanía, región conformada por 32 comunas y dividida administrativamente entre la Provincia de Malleco y la Provincia de Cautín, presenta antecedentes críticos respecto a niveles de crecimiento económico y desarrollo social. A nivel nacional, exhibe la mayor tasa de personas en situación de pobreza por ingresos; el ingreso más bajo; una de las más altas tasas de concentración de población mapuche a nivel nacional, y, la tasa de desempleo promedio supera el nivel nacional. 
Todos estos datos dan cuenta una región empobrecida económicamente y con carencia de espacios de reconocimiento intercultural.

Específicamente y en este contexto regional, la movilidad que nos interesa estudiar se denomina conmutación intrarregional, movimientos cotidianos de corta distancia que se dan principalmente por motivos de trabajo entre dos ciudades de una misma región. Es un hecho que las ciudades intermedias se caracterizan por generar relaciones de interacciones socioculturales, económicas y ecológicas con diferentes escalas, dando cuenta de un sistema de ciudades donde algunas son expulsoras y otras atractoras de flujos (Maturana Miranda \& Arenas Vásquez, 2012; Salazar et al., 2017a).

El caso de La Araucanía es interesante de destacar debido a su característica de intermediación, donde ciudades como Temuco, Angol y Villarrica son las principales atractoras de ocupados y estudiantes de las comunas aledañas (Salazar et al., 2017a). Asimismo, las transformaciones experimentadas por la capital regional durante la última década obligan a prestar atención a las diversas formas de segregación, contaminación ambiental y crecimiento de la pobreza como síntomas de una ciudad intermedia en pleno desarrollo (Marchant et al., 2016). Un último aspecto que destacar reside en los cambios en la estructura urbana de Temuco. La aceleración del crecimiento de población desde la década de los sesenta hace de la ciudad un fuerte polo de atracción para los habitantes de la misma región y del sur del país (Toledo et al., 2000).

El tema de la conmutación también ha sido tratado desde lo subjetivo, existiendo investigaciones que se han interesado en cómo los viajeros experimentan el viaje no solo focalizándose en las distancias y tiempos, sino que también, lo que implica en términos de organización, tácticas y experiencias. El punto central de muchas de estas investigaciones es indicar que las prácticas de conmutación son vividas como una pesada carga e implican estar constantemente cambiando y adaptándose a las circunstancias (Watts, 2008; Bissell, 2016; Vincent-Geslin \& Kaufmann, 2012).

Otra de las características de esta región es que a la oferta de transporte público existente, donde microbuses interurbanos y urbanos constituyen el grueso de dispositivos a disposición, se suma un cuarto medio: el ferrocarril de pasajeros. Dispositivo de transporte que a nivel nacional posee una baja frecuencia de circulación en comparación a otros dispositivos, reduciéndose exclusivamente a niveles regionales y con presencia en el Maule (Talca-Constitución), Biobío (Corto Laja y Biotren), Metropolitana (Nos) y Santiago-Rancagua. El ferrocarril de La Araucanía (Victoria-Temuco) se posiciona como el servicio más austral a nivel nacional.

Aunque el ferrocarril no es un medio de transporte masivo, posee características históricas, culturales y económicas que hacen que cobre un valor simbólico con fuerte arraigo en la cultura popular de la región. En la actualidad, el ramal Victoria-Temuco circula en 65 kilómetros a una velocidad de 50 kilómetros por hora, conectando las ciudades de Victoria, Perquenco, Lautaro y Temuco; y las localidades de P.U.A, Quillén y Pillanlelbún1.

Desde una perspectiva histórica, el ferrocarril en La Araucanía comenzó a funcionar desde fines del siglo XIX, permitiendo conectar la zona centro y la zona sur del país, también fue prioridad para vigilar y ejercer soberanía nacional en un territorio con históricos conflictos políticos y económicos con los mapuche de la zona. En la actualidad, la Empresa Ferrocarriles del Sur, concesionaría del servicio, traslada anualmente por la región a más de 500 mil personas (FESUR, 2016). La tarifa normal de un boleto Victoria-Temuco es $\$ 800^{2}$. El pasaje está subsidiado por el Estado de Chile. En la Figura 1 puede observarse el recorrido del ferrocarril por el territorio.

\footnotetext{
${ }^{1}$ Además, ofrece un servicio especial de lunes a viernes a los estudiantes del Instituto Claret.

${ }^{2}$ Aproximadamente 1,30 USD.
} 


\section{FERROCARRIL VICTORIA - TEMUCO | TIEMPO 90 MINUTOS}

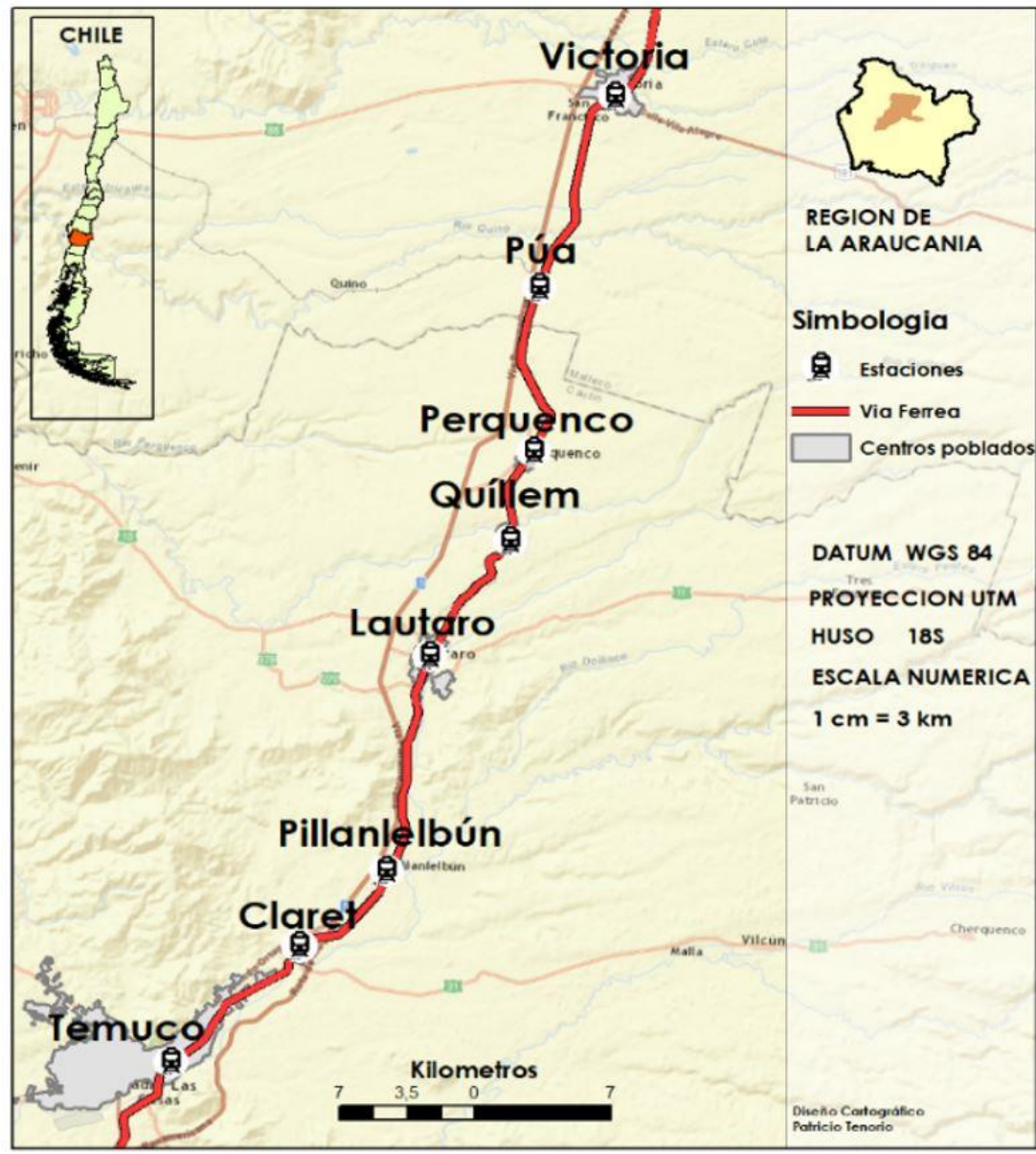

Figura 1 - Ruta del ferrocarril de La Araucanía. Fuente: Elaboración propia.

Finalmente, otro aspecto relevante de mencionar es que la Región de La Araucanía y las localidades donde habitan los sujetos estudiados se caracteriza por un elevado nivel de aislamiento, ruralidad y pobreza. Ello, hace que las localidades por donde pasa el ferrocarril estén expuestas a una doble dinámica: desigualdad y exclusión socio-espacial. En efecto, siguiendo a Jirón et al. (2010), la desigualdad la entenderemos como el proceso de posicionamiento, distribución y apropiación inequitativa de los recursos sociales, políticos, económicos y culturales entre los miembros de una sociedad, incorporando no sólo recursos materiales sino también recursos inmateriales o intangibles. Desde esta perspectiva, la exclusión socio-espacial tiene relación con las limitaciones de acceso y participación a dichos recursos y también a los recursos que ofrece la ciudad (Avellaneda \& Lazo-Corvalán, 2011), lo cual limita las posibilidades que ciertos habitantes de manera individual, tienen para insertarse en las dinámicas y procesos de desarrollo existentes. Sostenemos entonces que este proceso se da con intensidad en las localidades por donde pasa el ferrocarril y se diferencia de sus pares más urbanos que habitan en ciudades como Temuco. En este sentido, el conocimiento de las prácticas y experiencias de movilidad cotidiana de los sujetos, permite revelar tanto desigualdades a nivel local-regional como también al interior del mismo grupo.

\section{Enfoque metodológico: etnografías en movimiento y sombreos}

Dentro de los métodos que mayor interés han generado en los investigadores cualitativos, la etnografía cobra un papel fundamental. Para Guber (2001), la etnografía constituye un trabajo de campo 
flexible y abierto debido a que pone en el centro de análisis a los sujetos por sobre el investigador, permitiendo generar una interpretación de aspectos socioculturales para aquellos que no forman parte de espacios particulares de acción.

En Chile, durante los últimos años cobran relevancia metodologías móviles que centran sus análisis en las experiencias de los viajeros, combinando o utilizando diversas técnicas como la entrevista, la observación participante, los mapas mentales y el cuaderno de campo (Jirón et al., 2010; Lazo-Corvalán \& Calderón-Sosa, 2014, 2013; Imilan et al., 2015; Riquelme, 2017; Jirón \& Imilan, 2016; Salazar et al., $2017 \mathrm{~b}$, entre otros autores).

Una técnica que en los últimos años es utilizada con mayor frecuencia por los investigadores de la movilidad cotidiana se denomina sombreo (Jirón, 2011). El sombreo, tal como sugiere su nombre, consiste en convertirse en la sombra del viajero sirviendo de compañía en su rutina y desplazamientos por la ciudad, observando, preguntando, sintiendo y explorando los diversos momentos y movimientos que conforman el viaje, lo cual involucra en gran cantidad de ocasiones lugares que van más allá del medio de transporte y el viaje en sí, surgiendo la casa, el trabajo, parques, paraderos o caminatas, como lugares inmersos en las motivaciones de la movilidad.

En términos específicos ${ }^{3}$, y para este artículo, se presentan solo dos casos de habitantes de La Araucanía que por motivos laborales cotidianamente viajan. Luis (31 años) y Paula ${ }^{4}$ (32 años), fueron sombreados durante el invierno del año 2015, a partir de contactos establecidos en uno de los tantos viajes que se realizaron en el ferrocarril. Cabe señalar que hubo procedimientos previos y posteriores a la gestación del sombreo.

En primer lugar, y producto de una detenida y exhaustiva observación de los viajeros frecuentes ${ }^{5}$, Luis y Paula fueron divisados en reiteradas ocasiones en las estaciones del ferrocarril, lo cual los convirtió en potenciales participantes de la investigación. Posteriormente, fueron abordados con el objetivo de realizar una primera entrevista en profundidad. La entrevista fue realizada al interior de los vagones del ferrocarril y durante el viaje. En tercer lugar, y cuando ya existió un mayor vínculo humano y conocimiento de la investigación por parte de ambos, se propuso acompañarlos (sombrearlos) durante sus rutinas. Ambos accedieron, por ende, se planificó el viaje acordando el lugar de encuentro y duración del acompañamiento. Este ejercicio constituyó el primer ensayo de sombreo, el cual fue acompañado por una pauta de observación y un diseño de entrevista 6 . Ambos participantes, al momento de ser sombreados, trabajaban en una ciudad ajena a la de residencia; viajaban en ferrocarril hace dos años y vivían en compañía de sus familias.

\section{Moviéndose en ferrocarril: el trabajo en el centro y la ciudad transitoria}

A continuación, se presentan dos experiencias que constituyen una parte de los sombreos efectuados a dos usuarios del ferrocarril7. Ambos relatos reflejan movimientos, como también prácticas y experiencias de movilidad que develan dinámicas de inclusión y exclusión.

\footnotetext{
${ }^{3}$ Este artículo se enmarca en una investigación de doctorado (2012-2017) donde se trabajó con 22 personas que residen en una de las siete ciudades y localidades por donde transita el ferrocarril (Temuco, Lautaro, Victoria, Perquenco, P.U.A., Pillanlelbún y Quillén). Se sombrearon 14 personas y aquí se presentarán solo dos casos, los cuales se caracterizan por conectar diariamente distintas ciudades. ${ }^{4}$ Los nombres reales fueron cambiados con el objetivo de resguardar el anonimato.

${ }^{5}$ En principio, se viajó en el ferrocarril con el propósito de observar y distinguir los viajeros habituales de los viajeros esporádicos.

${ }^{6}$ Ambos insumos debieron someterse a la flexibilidad de las actividades que surgían en los acompañamientos, por lo cual, en algunos casos no llegaron a ser utilizados plenamente.

${ }^{7}$ Aunque se trabajó con 22 personas en el transcurso de una investigación más extensa que la aquí presentada, se escogieron sólo dos casos para presentar en este artículo a modo de experiencias de vida que pueden extrapolarse a otras similares y en sujetos que viven en lugares diferentes al que trabajan. En concordancia con el trabajo aquí exhibido, las experiencias de viajes por motivo de trabajo se tornaron un criterio de selección fundamental al momento de no incluir otras experiencias en este artículo.
} 


\section{"Acá la mayoría de la gente se mueve mucho en tren, nos cambió la vida"}

Luis es soltero y actualmente vive en Quillén junto su padre. Quillén es una localidad que administrativamente pertenece a la ciudad de Perquenco. Luis trabaja como albañil en la construcción, y para desarrollar su oficio durante su vida ha debido buscar trabajo en ciudades como Perquenco, Victoria y Temuco, ya que considera que en Quillén es difícil encontrar trabajo. En la Figura 2 se sintetiza el desplazamiento de Luis por distintos lugares de la región.

Ruta de desplazamiento de Luis Quíllem - Temuco

Datum - WGS 84

Proyección - UTM

Huso - 185

1. Casa de Luis - Estación de Quillem - 3 minutos

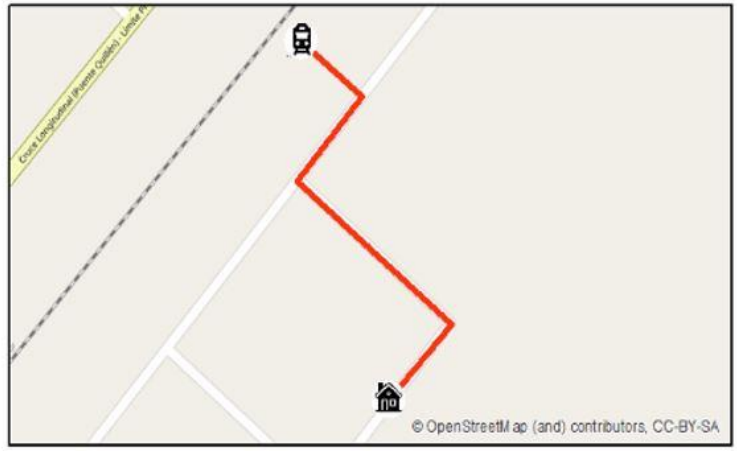

3. Estación de Temuco - Trabajo Luis - 25 minutos

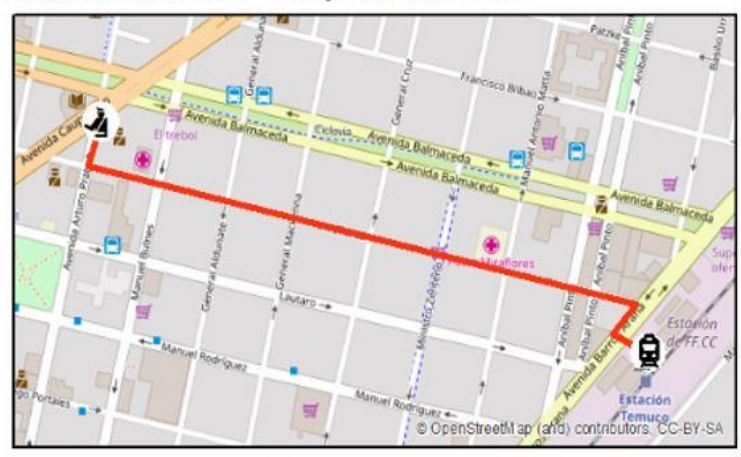

2. Estación de Quillem - Estación Temuco - 50 minutos

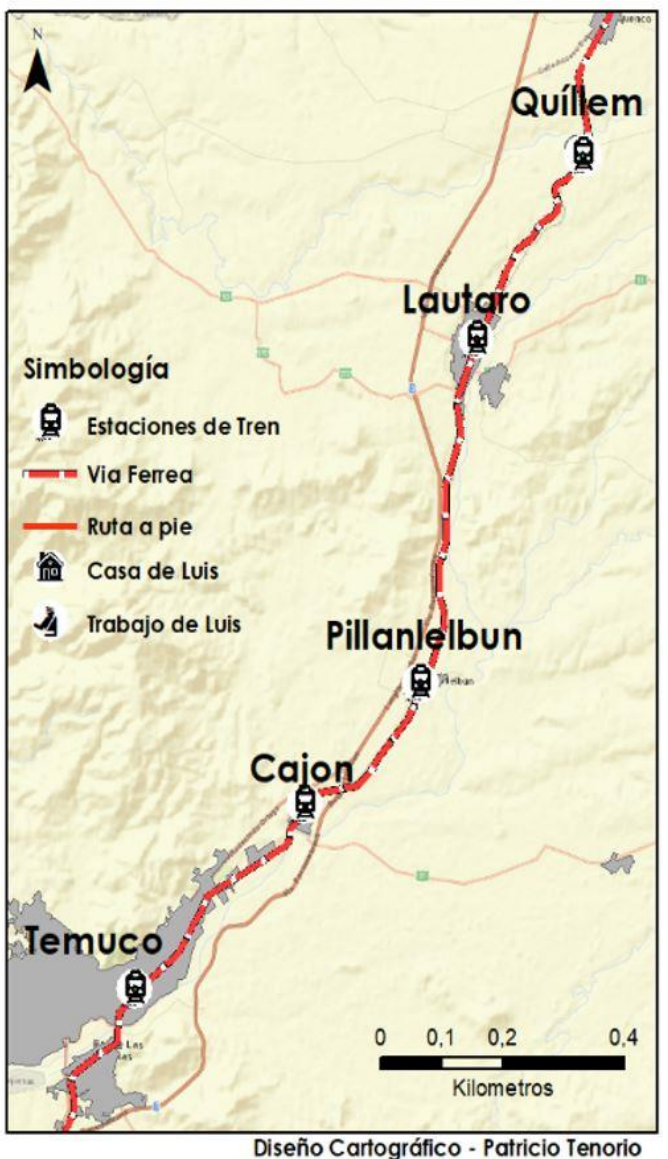

Figura 2 - Desplazamientos de Luis. Fuente: Elaboración propia.

Todos los días, Luis utiliza el ferrocarril para ir a trabajar. Se levanta poco antes de las 07:00 horas. Como Quillén comprende un territorio considerablemente reducido, calcula la hora de salida de casa rumbo a la estación con total perfección, no existen inconvenientes ni eventualidades que puedan retrasar su destino y no depende de otro medio que no sean sus pies para alcanzar el paradero. Son menos de 3 minutos de caminata los que separan su casa del paradero. Acompaño a Luis en el paradero de Quillén a la espera del ferrocarril de las 07.10 horas que nos llevará a Temuco. Junto a él hay otras personas aguardando, todos habitantes de Quillén que le devuelven el saludo y lo invitan a conversar mientras esperan el ferrocarril. Ya nos encontramos viajando, y apenas nos sentamos, comenta: "Acá la mayoría de la gente se mueve mucho en tren, nos cambió la vida. Si te fijas, mucho nos conocemos y eso hace el viaje más agradable".

El ferrocarril viaja con capacidad completa, Luis, sostiene que la mayoría va a trabajar a Temuco y en la noche retornan a sus hogares sólo para dormir, al día siguiente repiten lo mismo. Mientras viajamos revisa su celular y con una sonrisa manifiesta que la comodidad del tren es impagable. Estira sus piernas y se dispone a observar el paisaje. 
Para Luis, el paradero ferroviario de Quillén resulta de gran ayuda para la comunidad, ya que sin este medio de transporte difícilmente podrían encontrar conexión con otras comunas de la región. El motivo de ello se debe principalmente a que la localidad estuvo por mucho tiempo sin servicios públicos de transporte, por lo cual, sus habitantes debían acudir a la carretera a esperar algún bus. Luis recuerda que sólo pasaba una vez en la mañana y luego en la tarde, y ese era el principal medio de transporte para conectar con Perquenco. Actualmente se construyó un camino pavimentado por el interior de la localidad, y Luis señala que se ve mayor movimiento vehicular e inclusive ha realizado dedo para trasladarse, cuestión que antes no podía efectuar porque rara vez pasaban automóviles por el interior de Quillén, puesto que la mayoría de la locomoción privada y pública circula por la carretera.

El ferrocarril llega a Temuco poco antes de las 8 de la mañana, fueron 50 minutos de viaje. En Luis no se dejan ver rastros de cansancio, quizás un leve tedio por el frio que lo espera al descender del ferrocarril. Una vez en la estación de Temuco, Luis debe acudir a su lugar de trabajo, para tal efecto realiza un desplazamiento pedestre de 15 minutos con dirección oeste. Entra a trabajar a las 08:30 horas.

Caminamos hacia la salida de la estación. En la Feria Pinto, Luis acude a un carro de comida rápida donde compra una sopaipilla ${ }^{8}$ y un café, sostiene: “[...] este es mi desayuno casi todos los días. La señora de acá [la que atiende el local] ya me conoce y sabe con el hambre que vengo". Lo acompaño en su desayuno exprés, caminamos unas cuadras más y nos despedimos en la intersección de calle Lautaro con calle Prat con la finalidad de volver a encontrarnos por la tarde.

A eso de las 19:30 horas, nos volvemos a reunir en la estación de Temuco. Luis ha terminado su jornada laboral, viene cansado, con hambre y mojado producto de la fuerte lluvia que cae a esas horas. Mientras esperamos el servicio ferroviario, caminamos por la estación y conversamos de Quillén. Luis considera que Quillén es un poblado con una enorme dificultad de acceso y conexión, pero es un costo que asume con la mejor disposición, no obstante, los problemas que trae ello para la realización personal de sus habitantes es preocupante, sostiene: "[...] todo el pueblo usa el tren, los quinientos que somos, es como una salvación para nosotros desde que llegó, hace como dos o tres años, nos mejoró mucho la vida".

Para Luis, el servicio que ofrece la empresa de ferrocarriles es "[...] muy bueno, me deja salir de acá", no obstante, al no contar con un servicio los sábados o domingos por la mañana, debe reemplazar el viaje en ferrocarril por viajes en bus, situación que se traduce en un gasto económico importante. Si Luis por viajar en ferrocarril debe pagar $\$ 600$, por viajar en bus paga $\$ 1.000$.

En síntesis, para Luis el principal medio de transporte cotidiano es el ferrocarril, se va a Temuco por la mañana y vuelve a Quillén por la tarde en el servicio de las 20 horas, como segundo medio de transporte está el bus. En términos económicos y de comodidad el ferrocarril es fundamental.

El bus estresa mucho, que anda y que para, al final uno llega con la cabeza llena. En cambio, el tren tiene sus estaciones y nadie lo detiene hasta la otra estación. Uno ya conoce a los que viajan en el tren. Uno aprovecha de dormir un rato en el tren, en el bus uno no puede dormir.

Nos subimos al ferrocarril, poco alcanzamos a conversar. En no más de 10 minutos desde que partió, Luis ha cerrado los ojos para dormir profundamente. Sin más, me voy a su lado viendo como la mayoría de los viajeros presenta una actitud similar, una mezcla de cansancio y ansiedad por llegar luego a sus casas, mientras de fondo el sonido del tren y la lluvia que corre por las ventanas reflejan una parte del paisaje de la región.

Luis despierta en Lautaro, luego de 40 minutos de viaje, sabe que queda sólo una estación antes de llegar a Quillén. Aprovecha de ir al baño, comenta: “[...] estiro las piernas un ratito, me acomodo y vuelta a echar la última pestañáa". La actitud de cansancio es la misma, hablamos un poco de fútbol pero el sueño lo supera y vuelve a dormirse. Automáticamente, casi como si supiera dónde debe bajarse sin necesidad de estar despierto, abre los ojos minutos antes de llegar al paradero de Quillén. Son casi las 20:40 horas y Luis se dispone a bajar. Nos despedimos en el mismo ferrocarril. Yo sigo hacia Victoria.

\footnotetext{
${ }^{8}$ Masa frita.

${ }^{9}$ Siesta breve.
} 
"El tren pasa por dentro de Pillanlelbún a diferencia de los buses que tengo que tomarlos en la carretera"

Paula, nació y actualmente vive junto a su madre y su hermano en la Villa Ultra Cautín de Lautaro. Estudió Trabajo Social y actualmente trabaja en un Liceo de Pillanlelbún, localidad que pertenece a Temuco. En la Figura 3 se presenta la rutina de Paula en base a sus desplazamientos cotidianos.

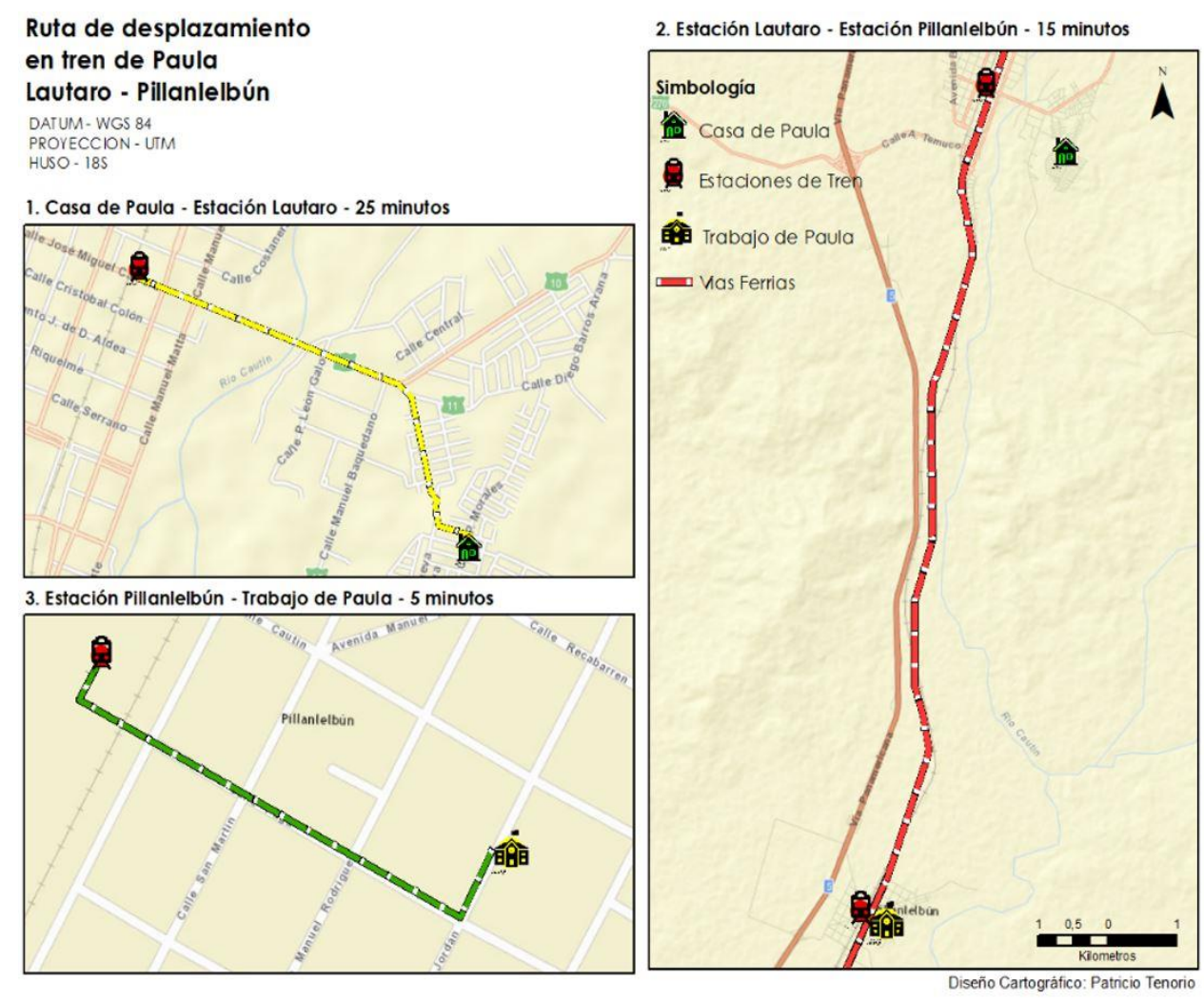

Figura 3 - Desplazamientos de Paula. Fuente: Elaboración propia.

Paula, de lunes a viernes, se despierta a eso de las 06.45 horas para acudir a trabajar, luego de levantarse y desayunar sale de su casa para comenzar su jornada habitual. Lo primero que hace es caminar una cuadra para tomar el colectivo que la acercará a la plaza principal. La elección de colectivo ${ }^{10}$, en desmedro del micro, se debe a la comodidad y el ahorro de tiempo, a pesar de que el pasaje del colectivo cuesta $\$ 250$ (\$50 más que el micro), la costumbre y la comodidad la han transformado en usuaria habitual del colectivo.

Han pasado 20 minutos desde que salió de casa y está a la espera del microbús de las 07:45 horas que la llevará a Pillanlelbún (ya está al tanto de los horarios, por lo cual, siempre toma el mismo). Paula, en gran cantidad de los viajes que realiza en la mañana, opta por el microbús intercomunal ya que el ferrocarril la dejaría muy temprano en el colegio:

Entro a trabajar a eso de las 08:30 y el tren está en Pillanlelbún a las 07:30, entonces para qué llegar una hora antes a trabajar. Hay veces donde lo hago, cuando tengo que estar muy temprano, pero como yo hay mucho otros a los que no les sirve mucho el horario de la mañana.

El microbús va repleto de pasajeros, razón por la cual debe viajar de pie, me dice: “[...] menos mal que en el primer viaje me toca parada ; a la vuelta sería horrible!”. Hacia Pillanlelbún no van muchos, tanto así que hoy es la única en bajar. El viaje del micro con dirección a Temuco no incluye en su ruta el interior de

${ }^{10}$ El colectivo es un medio de transporte público urbano que es compartido entre diversos usuarios (con capacidad máxima de cuatro pasajeros). 
la comuna, producto de ello debe bajarse en la carretera y caminar 15 minutos para llegar a Pillanlelbún. Según Paula, hace varios años que el microbús dejó de pasar por dentro y ello supone un aislamiento de la comuna con el resto de la región, a diferencia del ferrocarril que sí tiene un paradero cercano al centro. La caminata de la carretera a Pillanlelbún es descrita por Paula de la siguiente manera: "[...] para ingresar hay dos pasarelas, la peatonal y la de vehículos, yo generalmente voy por la peatonal, por el clima y la luz, pero en invierno era más riesgoso por la oscuridad".

Una vez que ha llegado al liceo, a eso de las 08:25 horas, comienza sus labores profesionales. Su jornada laboral concluye a las 17:00 horas. La organizó de esta manera para retornar a su hogar desplazándose en ferrocarril. En un principio trabajaba hasta las 17.30 horas, pero sus colegas le informaron que existía la posibilidad de retornar en ferrocarril, específicamente en el servicio de las 17:07 horas. Otros factores que la impulsaron a utilizarlo quedan reflejados de la siguiente manera:

Comodidad, porque me sale mucho más barato el pasaje, y la razón principal es que el tren pasa por dentro de Pillanlelbún a diferencia de los buses que tengo que tomarlos en la carretera. Me gusta mucho el ambiente que se da en el tren, he conocido gente en el tren de repente conversando, siento que es un ambiente distendido a diferencia del bus que es más individualista.

El viaje de Pillanlelbún a Lautaro tiene un costo de $\$ 450$, mientras que un pasaje en autobús equivale a $\$ 600$. Mientras viaja observa el paisaje, lee y escucha música, y en algunas ocasiones conversa con otros usuarios, sostiene:

[...] a veces, cuando me siento al lado de alguien, me preguntan: ¿Dónde vas? ¿Cuál es la próxima estación?, y de ahí se da interacción con más personas. A veces pasa que con un desconocido se genera un ambiente rico de conversación.

Lautaro es la comuna donde ha residido gran parte de su vida. Hace 15 años que vive al otro lado de la ciudad, específicamente atravesando el rio Cautín, es uno de los barrios más antiguos, y los costos de vivir en la periferia de la ciudad, según ella, impactan en la accesibilidad a servicios como supermercados, bancos, correos y comercio variado. En su barrio sólo hay almacenes pequeños y casas. Luego de caminar un par de cuadras desde el liceo al paradero, esperamos el tren de las 17:07 horas rumbo a Lautaro. Mientras viajamos me comenta que durante sus estudios universitarios vivió en Temuco, ciudad que le generaba estrés debido al ritmo de vida. Lautaro siempre estuvo presente para ella, siempre pensó en retornar, lo define como un lugar que llama a volver. Paula observa el paisaje y comenta:

Lo que me gusta de viajar en tren, a diferencia de viajar en bus, es que me siento más tranquila y reflexiono de mi vida ;Algo importante es el paisaje!, por ejemplo, cuando viajo en micro veo sólo casas y casas, y en tren veo ríos, bosques y la cantera, que es un lugar que está entre Lautaro y Pillanlelbún.

Hoy el ferrocarril lleva consigo un número importante de pasajeros, el viaje no tarda más de 20 minutos y ya estamos dispuestos para bajar en el paradero Lautaro centro. Una vez que llegamos, Paula, se dispone a caminar hacia la plaza principal, donde esperará el colectivo número 1 . Desciende del ferrocarril y al pensar en llegar a su casa comenta: "[...] ahora viene la vuelta, tengo que caminar para tomar el colectivo, atravesar el puente, bajar del colectivo y caminar ¿cachai que todavía me queda viaje? Cuando me bajo del tren me viene el cansancio y quiero puro llegar a la casa".

Hoy volverá a casa para descansar, no tiene ninguna actividad en mente. Como hoy camina hacia su casa, la acompaño y mientras avanzamos me indica el paradero del microbús, el paradero del colectivo y las calles por donde transita frecuentemente. Una vez en el puente, comenta: "[...] el puente divide la ciudad, para allá [atravesando el puente] vivo yo ¿te dai cuenta que son más casas nomás? Si queremos salir de la población tenemos que atravesar el puente". Nos despedimos y quedamos de juntarnos en la semana para hacernos compañía durante el viaje nuevamente. 


\section{Conclusiones: el ferrocarril como mecanismo de inclusión socioespacial}

Un motivo fundamental que ha conllevado a pensar la movilidad por motivos laborales reside en la lejanía que presenta el lugar de trabajo respecto al lugar de residencia (Susino \& Martínez, 2010), lo que algunos autores han llamado conmutación intrarregional. En tal sentido, los casos de Luis y Paula reflejan prácticas de movilidad propias de las ciudades y poblados del sur de Chile (Salazar et al., 2017a), donde sus habitantes residen y trabajan en ciudades distintas, y por lo tanto muchas veces deben realizar largos y pesados desplazamientos. Aunque este artículo muestra dos casos enmarcados en una investigación de mayor extensión, se advierte que en el resto de los casos pesquisados las experiencias de movilidad abordaron también otras temáticas vinculadas al género, segregación residencial y movilidad estudiantil. Estos casos presentan problemáticas que escapan de lo netamente ligado a la conmutación aquí presentada.

Paula, habitante de una ciudad pequeña pero con circuitos de transporte medianamente eficientes, reside en un barrio alejado del centro urbano de Lautaro, el cual presenta deficiente conectividad y escasa oferta de servicios. Los viajes desde Lautaro a Pillanlelbún involucran una alta espesura (Jirón \& Mansilla, 2014) en lo que respecta a las distancias y tiempos empleados en la movilidad. De sus experiencias de viaje se desprenden barreras de accesibilidad que se agudizan producto de vivir en un barrio periférico y trabajar en Pillanlelbún, localidad con deficiente disponibilidad de medios de transporte público.

Luis, habitante de una localidad que presenta una conectividad altamente reducida en términos de dispositivos de transporte a disposición, cuando realiza los viajes con destino a Temuco comienza a percibir barreras a medida que intenta acceder a servicios. Si bien Luis no cubre largos desplazamientos para acceder al ferrocarril ya que su lugar de residencia está a unos pocos metros del paradero de ferrocarril, manifiesta barreras a nivel de acceso a espacios de recreación, trabajo y educación. En Quillén no hay mucho más que casas, algún boliche y un rudimentario centro de salud.

En las experiencias cotidianas de ambos se pueden encontrar interesantes esfuerzos por garantizar su presencia en los respectivos lugares de trabajo. Paula y Luis han incorporado el ferrocarril como medio de transporte fundamental de entrada y salida, por ende, de conexión entre los lugares, y ello resulta fundamental, puesto que permite evitar desplazamientos pedestres que debiesen hacer si es que utilizaran buses interurbanos, ya que a diferencia de estos el ferrocarril circula por el interior de Quillén y Pillanlelbún. Ambos han organizado sus horarios laborales en función de los horarios del ferrocarril por razones de acceso, y otras como el costo del pasaje, una experiencia de viaje más ameno y, sobre todo en el caso de Luis, por ser el único medio de transporte público que le permite entrar y salir de la localidad sin tener que caminar a la carretera.

La relevancia del entorno, en ambos casos, permite demostrar la disminución de la calidad de vida producto de no contar con opciones de transporte que fomenten la comunicación con otros espacios y lugares, por lo cual, el déficit de equipamiento en sus lugares de residencia conlleva a que deban salir de sus ciudades para así participar activamente en la vida social pública.

El ferrocarril constituye un lugar relevante en las interacciones sociales y experiencias cotidianas de los usuarios. La elección de este dispositivo de transporte no solo se da por aspectos vinculados a la oferta de servicios, sino que también se relaciona a los positivos atributos subjetivos que le son otorgados como lugar transitorio, instancia de sociabilidad y encuentro (Jirón et al., 2010). Vivir en regiones no metropolitanas conlleva a pensar la movilidad desde las particularidades del territorio, produciendo en muchas ocasiones la diminución de posibilidades de acceso, gran cantidad de viajes y largas horas de desplazamiento. En este sentido son las prácticas, las tácticas y las estrategias de los usuarios las que permiten sortear las dinámicas de exclusión propias de este tipo de territorios.

Por otra parte, y en términos objetivos, no todos optan a los mismos medios de transporte y los criterios de selectividad dependerán de factores sociales que inciden en las experiencias de viaje de cada sujeto. Asimismo, y en términos subjetivos, no todos tienen la misma experiencia del viaje y la viven de la misma manera, a pesar de viajar en el mismo medio de transporte. En este sentido, la movilidad nunca es igual y va cambiando a los sujetos por medio de las prácticas cotidianas. 
Finalmente, a través de los casos presentados se evidencia la importancia de conocer la experiencia de la movilidad para entender las dinámicas de inclusión y exclusión socioespacial. Cobra relevancia no sólo el lugar frecuentado, sino que también cómo las personas experimentan a diario el movimiento, así como las prácticas y las estrategias que la movilidad conlleva. A partir de este estudio, fue posible revelar dinámicas de inclusión y de exclusión propias de territorios rezagados como el que aquí se presentó, donde muchas veces el espacio vivido se tensiona con la ciudad planificada. En el contexto actual, la movilidad juega un rol importante no solo a gran escala sino también en la vida cotidiana de los individuos que viven en estas pequeñas localidades aisladas. En este escenario, una parte importante de las actividades cotidianas de los sujetos estudiados requiere de desplazamientos dependientes y posibles producto de dispositivos como el ferrocarril. En rigor, la movilidad debe entenderse como algo más que un mero desplazamiento; la movilidad debe comprenderse como una experiencia y a la vez como un importante capital social en tanto permite el acceso de los sujetos a una red de relaciones sociales, conocimientos y territorios distintos, imprescindibles para el desarrollo de su vida cotidiana.

\section{Referencias}

Aguiar, S. (2011). Dinámicas de la segregación urbana: movilidad cotidiana en Montevideo. Revista de Ciencias Sociales, (24), 55-76.

Avellaneda, P. (2008). Movilidad cotidiana, pobreza y exclusión social en la ciudad de Lima. Annales de Géographie, 28(2), 9-35.

Avellaneda, P., \& Lazo-Corvalán, A. (2011). Aproximación a la movilidad cotidiana en la periferia pobre de dos ciudades latinoamericana: los casos de Lima y Santiago de Chile. Revista Transporte y Territorio, 4, 47-58.

Bissell, D. (2016). Micropolitics of mobility: public transport commuting and everyday encounters with forces of enablement and constraint. Annals of the Association of American Geographers, 106(2), 394-403.

Castells, M. (1973). La urbanización dependiente en América Latina. In M. Castells (Ed.), Imperialismo y urbanización en América Latina (pp. 7-26). Barcelona: Gustavo Gili.

Cortés, S. (2011). Al infinito, y más allá. Espacialidad y movilidad en la vida cotidiana de niñas y niños en Santiago, Chile (Tesis de pregrado). Universidad de Chile, Santiago de Chile.

Cosacov, N. (2015). Más allá de la vivienda: los usos de la ciudad: movilidad cotidiana de residentes en Buenos Aires. Estudios Socioterritoriales, (18), 61-80.

Cresswell, T. (2006). On the mover. London: Routlege.

Cresswell, T. (2010). Towards a politics of mobility. Environment and Planning: Society and Space, 28(1), 17-31. Recuperado el 14 de febrero de 2018, de http://journals.sagepub.com/doi/abs/10.1068/d11407

Errázuriz, T. (2012). Para una historia del viaje metropolitano. Bifurcaciones, (11), 1-13.

Fagerholm, N., \& Broberg, A. (2011). Mapping and characterising children's daily mobility in urban residential areas in Turku, Finland. Fennia, 189(2), 31-46.

Ferrocarriles del Sur - FESUR. (2016). Reporte de sostenibilidad. Concepción: Ferrocarriles del Sur. S.A.

Guber, R. (2001). La etnografía: método, campo y reflexividad. Bogotá: Grupo Editorial Norma.

Gutiérrez, A. (2009). La movilidad de la metrópolis desigual: el viaje a la salud pública y gratuita en la periferia de Buenos Aires. In Actas del XII Encuentro de Geógrafos de América Latina. Montevidéu: Universidad de la República de Uruguay.

Harvey, D. (2012). Rebel cities: from the right to the city to the urban revolution. London: Verso.

Imilan, W., Jirón, P., \& Iturra, L. (2015). Más allá del barrio: habitar Santiago en la movilidad cotidiana. Revista Antropologías del Sur, (3), 87-103. 
Instituto Nacional de Estadísticas - INE. (2016). Demográficas y vitales. Chile. Recuperado el 14 de febrero de 2018, de http://www.ine.cl/estadisticas/demograficas-y-vitales

Jirón, P. (2007). Implicancias de género en las experiencias de movilidad cotidiana urbana en Santiago de Chile. Revista Venezolana de Estudios de la Mujer, 12(29), 173-198.

Jirón, P. (2011). On becoming la sombra/the shadow. In M. Buscher, J. Urry, \& K. Witchger (Eds.), Mobile methods (pp. 36-53). London: Routledge.

Jirón, P., \& Imilan, W. (2016). Observando juntos en movimiento: posibilidades, desafíos o encrucijadas de una etnografía colectiva. Alteridades, 26(52), 51-64.

Jirón, P., \& Mansilla, P. (2014). Las consecuencias del urbanismo fragmentador en la vida cotidiana de habitantes de la ciudad de Santiago. EURE. Revista Latinoamericana de Estudios Urbano Regionales, 40(121), 5-28. http://dx.doi.org/10.4067/S0250-71612014000300001.

Jirón, P., \& Zunino, D. (2017). Dossier: movilidad urbana y género: experiencias latinoamericanas. Revista Transporte y Territorio, (16), 1-8.

Jirón, P., Lange, C., \& Bertrand, M. (2010). Exclusión y desigualdad espacial: retrato desde la movilidad cotidiana. Revista INVI, 25(68), 15-57. http://dx.doi.org/10.4067/S0718-83582010000100002.

Jouffe, Y., \& Lazo Corvalán, A. (2010). Las prácticas cotidianas frente a los dispositivos de la movilidad: aproximación política a la movilidad cotidiana de las poblaciones pobres periurbanas de Santiago de Chile. EURE. Revista Latinoamericana de Estudios Urbano Regionales, 36(108), 29-47. http://dx.doi.org/10.4067/S025071612010000200002.

Landon, P. (2013). Movilidad cotidiana y exclusión social: anverso y reverso de la instalación de la autopista acceso sur en la periferia pobre de la metrópolis de Santiago de Chile. Lima: Congreso Tercer EIMUS.

Lazo-Corvalán, A., \& Ther, F. (2014). L'univers du voyage: géographie matérielle et sensorielle des mobilités dans l'archipel de Quinchao, Chiloé, Chili. Géographie et Cultures, 91(92), 49-64. http://dx.doi.org/10.4000/gc.3327.

Lazo-Corvalán, A., \& Carvajal, D. (2018). Habitando la movilidad: El viaje en lancha, los objetos y la experiencia de la movilidad en el archipiélago de Quinchao, Chiloé. Revista Austral de Ciencias Sociales, 33, 89-102.

Lazo-Corvalán, A., \& Calderón-Sosa, R. (2013). Proximidad y movilidad en la metrópolis contemporánea: etnografía de tres barrios de la ciudad de Santiago de Chile. Revista Bitácora Urbano Territorial, 22(1), 89-96.

Lazo-Corvalán, A., \& Calderón-Sosa, R. (2014). Los anclajes en la proximidad y la movilidad cotidiana: Retrato de tres barrios de la ciudad de Santiago de Chile. EURE. Revista Latinoamericana de Estudios Urbano Regionales, 40(121), 121-140. http://dx.doi.org/10.4067/S0250-71612014000300006.

Lefebvre, H. (1975). El derecho a la ciudad. Barcelona: Península.

Marchant, C., Frick, J., \& Vergara, L. (2016). Urban growth trends in midsize Chilean cities: the case of Temuco. Urbe. Revista Brasileira de Gestão Urbana, 8(3), 375-389. http://dx.doi.org/10.1590/2175-3369.008.003.A007.

Maturana Miranda, F. R., \& Arenas Vásquez, F. (2012). El policentrismo en Chile: medición exploratoria para el sistema de ciudades de las regiones de La Araucanía, de Los Ríos y de Los Lagos. Revista de Geografía Norte Grande, (52), 37-56. http://dx.doi.org/10.4067/S0718-34022012000200003.

Ramírez, B. (2013). Nuevo paradigma o cambios en la territorialidad de la movilidad, una reflexión teórica (pp. 373416). México: UAM.

Ribera Fumaz, R., Vivas, P., \& González, F. (2007). Ciudades en la sociedad de la información: una introducción. UOC Papers, (5), 1-36.

Riquelme, H. (2017). Cruzando La Araucanía (Chile): un análisis de las prácticas de movilidad cotidiana de universitarios adscritos a la gratuidad. Cultura-hombre Sociedad, 27(1), 23-50. http://dx.doi.org/10.7770/cuhsoV27N1-art1131. 
Rolnik, R., \& Klintowitz, D. (2011). (I)mobilidade na cidade de São Paulo. Estudos Avançados, 25(71), 89-108. http://dx.doi.org/10.1590/S0103-40142011000100007.

Salazar, G., Irarrázaval, F., \& Fonck, M. (2017a). Ciudades intermedias y gobiernos locales: desfases escalares en la Región de La Araucanía, Chile. EURE. Revista Latinoamericana de Estudios Urbano Regionales, 43(130), 161-184. http://dx.doi.org/10.4067/s0250-71612017000300161.

Salazar, G., Fonck, M., \& Irarrázaval, F. (2017b). Paisajes en movimiento: Sentidos de lugar y prácticas interculturales en ciudades de la Región de La Araucanía, Chile. Chungara, 49(2), 251-264.

Schwanen, T., \& Páez, A. (2010). The mobility of older people: an introduction. Journal of Transport Geography, 18(5), 591-595. http://dx.doi.org/10.1016/j.jtrangeo.2010.06.001.

Segura, R. (2006). Segregación residencial, fronteras urbanas y movilidad territorial: un acercamiento etnográfico (Cuadernos del IDES, No. 9). Buenos Aires: IDES.

Sheller, M., \& Urry, J. (2006). The new mobilities paradigm. Environment \& Planning A, 38(2), 207-226. http://dx.doi.org/10.1068/a37268.

Simmel, G. (2005). La metrópolis y la vida mental. Bifurcaciones, (4), 1-10.

Singer, P. (1975). Economía política de la urbanización. México: Siglo XXI.

Soto, P. (2017). Diferencias de género en la movilidad urbana. Las experiencias de viaje de mujeres en el Metro de la Ciudad de México. Revista Transporte y Territorio, (16), 127-146.

Stjernborg, V. (2017). The meaning of social participation for daily mobility in later life: an ethnographic. Case study of a senior project in a swedish urban neighbourhood. Ageing International, 42(3), 374-391. http://dx.doi.org/10.1007/s12126-017-9296-4. PMid:28890586.

Susino, J., \& Martínez, J. (2010). Movilidad cotidiana por trabajo en el análisis de la estructura territorial: Andalucía y centro de México. Papeles de Población, 16(66), 137-176.

Toledo, X., Romero, H., \& Garín, A. (2000). Segregación socioespacial de la comuna de Temuco. Espacio y Desarrollo, (12), 103-122.

Urry, J. (2007). Mobilities. Cambridge: Polity.

Vincent-Geslin, S., \& Kaufmann, V. (2012). (Eds.). Mobilité sans racines: plus loin, plus vite... plus mobiles. Paris: Descartes et Cie, Cultures Mobiles.

Watts, L. (2008). The art and craft of train travel. Social \& Cultural Geography, 9(6), 711-726. http://dx.doi.org/10.1080/14649360802292520.

\section{Editor: Fábio Duarte}

Recibido: Feb. 14, 2018

Aprobado: Ago. 05, 2018 